\title{
Recent Trends in Physique and Motor Ability of Preschool Children-Trends After 2000 in Japan
}

\author{
Kohsuke Kasuya ${ }^{1, ~ *, ~ K a t s u n o r i ~ F u j i i ~}{ }^{1}$, Nozomi Tanaka ${ }^{2}$, Toshiro Sakai ${ }^{3}$, Yuki Takeyama ${ }^{1}$ \\ ${ }^{1}$ Graduate School of Business Administration and Computer Science, Aichi Institute of Technology, Toyota, Aichi, Japan \\ ${ }^{2}$ Sports and Health Science, Tokai Gakuen University, Miyoshi, Aichi, Japan \\ ${ }^{3}$ Life and Health Science, Chubu University, Kasugai, Aichi, Japan \\ Email address: \\ kohsuke28kasuya@gmail.com (K. Kasuya), fujii@aitech.ac.jp (K. Fujii), tanaka-n@tokaigakuen-u.ac.jp (N. Tanaka), \\ sakai@ipc.chubu.ac.jp (T. Sakai), yuki319takeyama@yahoo.co.jp (Y. Takeyama) \\ ${ }^{*}$ Corresponding author
}

\section{To cite this article:}

Kohsuke Kasuya, Katsunori Fujii, Nozomi Tanaka, Toshiro Sakai, Yuki Takeyama. Recent Trends in Physique and Motor Ability of Preschool Children-Trends After 2000 in Japan. American Journal of Sports Science. Vol. 8, No. 2, 2020, pp. 33-38. doi: 10.11648/j.ajss.20200802.12

Received: May 14, 2020; Accepted: June 1, 2020; Published: June 17, 2020

\begin{abstract}
The purpose of this study was to clarify the trends since 2000 in body shape, physical strength, and motor ability in early childhood in Japan. The study subjects were kindergarten and nursery school girls (age 3-5 years old) in Aichi Prefecture, Japan. Physique (height and weight), quantitative motor ability (20 m dash, standing long jump, tennis ball throw, side step, one-leg hop, hanging from a horizontal bar, and general motor ability (jump over and under) were compared in the 1999 and 2009 school years. The results revealed that, compared with ten years earlier, height was approximately $0.9 \mathrm{~cm}$ shorter in $4-y e a r-o l d$ girls and weight was approximately $0.3 \mathrm{~kg}$ lighter in 3- and 4-year-old girls in 2009. In physical strength and motor ability, the time for jump over and under was shorter in 3-year-olds, the number of times a rope was jumped was higher and the time hanging from a horizontal bar was longer, and the time for jump over and under was shorter in 4-year-olds. In 5-year-olds, only an increase in the number of times a rope was jumped increased. The tennis ball throw and side steps were not significantly different from 10 years earlier in any of the ages. Physique, physical strength, and motor ability improved with growth, but compared with 10 years earlier many of the items were found to decrease or remain the same in all ages.
\end{abstract}

Keywords: Preschool Girls, Physique, Physical Strength, Annual Comparison

\section{Introduction}

More than 20 years have passed since Windows 95 was launched in Japan, and the computer age has truly arrived. In recent years especially, the number of Internet-addicted children has been rapidly increasing world-wide together with the spread of smart phones. As a result, "smartphone cognitive impairment" has come to be viewed as a problem in young people. The computer age is depriving people of physical activity and new diseases are emerging from disruption in the balance of living activities. Ultimately, Japan's high economic growth has resulted in acceptance of a society that overemphasizes intellectual development, resulting in the loss of outdoor play for children and a lack of free time to develop physically. Nishijima's [1] well-known examination of the decreased physical fitness of children found that the physical strength and motor ability of children today is quite poor relative to their body size. Particularly low were physical functions and abilities that do not appear in the results of physical strength measurements, for example activities and motor performance that occur in actual daily life, so that it is becoming more common for children to abandon tasks before completion or suffer accidents and injuries. There is also thought to be a risk of losing the "inner potential for growth" to live as a human. According to Ueda [2], the inner potential for growth is the change in the meaning of life caused by the energy of internal time variations that come from inside humans.

A recent physical fitness and motor ability survey report [3] by the Ministry of Education, Culture, Sports, Science and Technology indicated that while physical fitness does not reach the level of that in 1985, the decline has stopped. Even though the decline has stopped, however, there has not been a 
return to past levels and physical strength is without question low relative to body size. However, do low levels of strength mean that one cannot survive in modern society? Hayakawa and Fujii [4-6] proposed the concept of valid health and physical fitness, which implicates the importance of having physical fitness suited to one's living behaviors for modern humans. In that sense, is children's physical fitness low in an absolute sense? Nishijima [1] conducted an examination for school age children, but for preschool children it is not in fact clear that levels are low.

Akimaru et al. [7] and Akimaru [8] compared the physical fitness results of preschool children from the changes over time in the 30 years between 1969 and 1999, and showed that while in some activities there was a decreasing trend, in others there was an increasing trend. This suggested that, depending on the activity, there were in fact some rising trends among preschool children. Why would secular trends in physical fitness and motor ability differ between preschool children and schoolchildren? One may point to the phenomenon of earlier maturation in the growth and development of physique and motor ability. When physical maturation happens sooner in early childhood through normal mechanisms, it is predicted that as the body becomes bigger motor ability equal to that growth is acquired or improved. Fujii et al. [9-11] examined the increasingly early physical maturation of young children and indicated that in fact there were rising trends in tests of motor ability in young children except for in throwing. Thus, although motor ability obviously improves as the body matures, the increase from earlier maturation is canceled out by the declining trend in motor ability. This explains the ambiguity in the trend for declining motor ability in young children. Kindergartens are said to be the educational setting with the highest degree of freedom, and Kasuga [12] explained the huge effect of kindergarten environment on physical fitness in young children, with the level of physical fitness of kindergarten children differing depending on the kindergarten they attend.

This study investigated the trends in physical ability of young children in recent years by comparing the physical fitness and motor ability results in the two years of 1999 and 2009 , to examine the question of whether or not the physical abilities of young children have actually deteriorated in the years since 2000 .

\section{Methods}

\subsection{Subjects}

The subjects were girls from several dozen kindergartens in Aichi Prefecture, Japan. Measurements of physique and motor ability of 3-, 4-, and 5-year-old girls in 1999 and 2000 were used in this study. Girls were taken as the subjects because of the difference in the dynamics of growth and development between boys and girls. As shown in Table 1, the number of girls measured in each activity differed. Before making the measurements, the content of the measurements and the handling of the measurement data were fully explained to the girls, their parents, and the kindergarten teachers, and their consent to participate in the measurements was obtained.

\subsection{Measurements}

As an indicator of physique, height and weight were measured and body mass index (BMI) was calculated by dividing weight $(\mathrm{kg})$ by the square of height $(\mathrm{cm})$. The motor ability items were the $20 \mathrm{~m}$ dash, standing long jump, tennis ball throw, side step, one-leg hop, hanging from a horizontal bar, and jump over and under. The measurements were performed following the motor ability measurements described in Akimaru et al. [7] and Akimaru [8]. The measurement methods are described in Figures 1-7 and below.

\subsubsection{0m Dash}

A straight $20 \mathrm{~m}$ course was set up. A false goal line was set up $3 \mathrm{~m}$ beyond the real goal line, and the girls were instructed to run as fast as they could to the false goal line. Pairs of girls of roughly the same speed ran together, and the race started when teachers standing behind the runners gently pressed them on the back at a signal from the starter. The time was measured until some part of the runner's body reached the goal line. The measurements were made one time only. Measurements were avoided on days with strong wind.

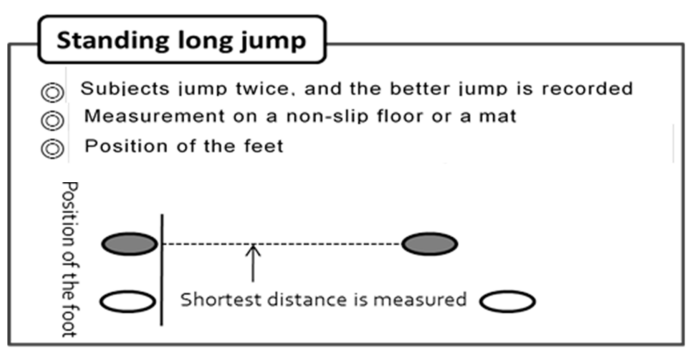

Figure 1. Method of measuring $20 \mathrm{~m}$ dash.

\subsubsection{Standing Long Jump}

The measurements were made on a mat or a non-slip floor. The subjects were asked to jump with both legs, and the shortest distance from toe at the start line to the heel after the jump was recorded. Subjects jumped twice, and the better of the two jumps was recorded.

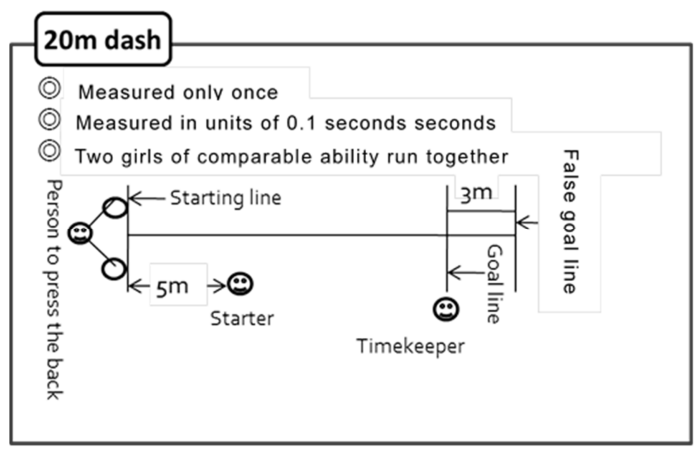

Figure 2. Method of measuring standing long jump. 


\subsubsection{Tennis Ball Throw}

A regular tennis ball was used. The girls stood at the measurement line, and the distance the ball was thrown was measured in units of centimeters with a tape measure stretched along the ground. The ball was thrown twice consecutively, and the better of the two throws was recorded.

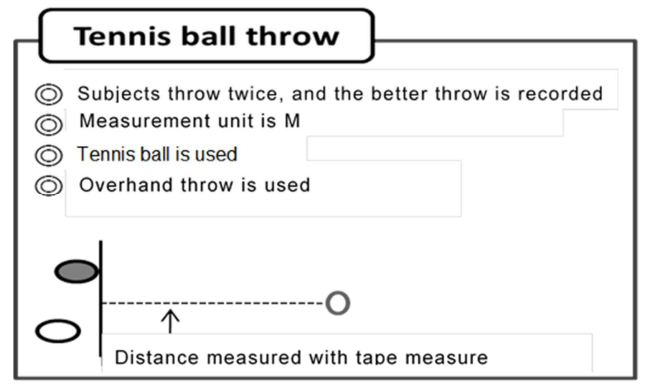

Figure 3. Method of measuring tennis ball throw.

\subsubsection{One-leg Hop}

On a straight line with a distance of $20 \mathrm{~m}$ one way, subjects hopped on one leg up and back. The total distance they could continue on one leg was recorded in meters. If they lost their balance so that the other foot touched the ground, they were told they could continue but not to switch legs. Pairs of girls though to have about the same ability were measured together a single time. They were stopped when they had hopped more than 50 meters.

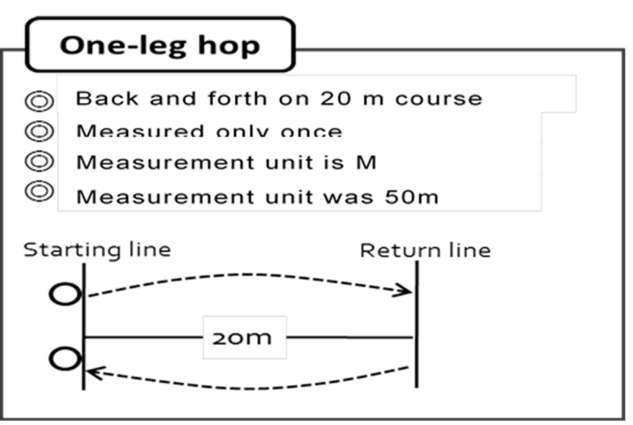

Figure 4. Method of measuring one-leg hop.

\subsubsection{Jump over and Under}

A rope was stretched $30 \mathrm{~cm}$ above the ground, and subjects were instructed to jump over the rope and then crawl back under. The number of seconds it took to perform a fixed number of over-and-under movements was recorded.

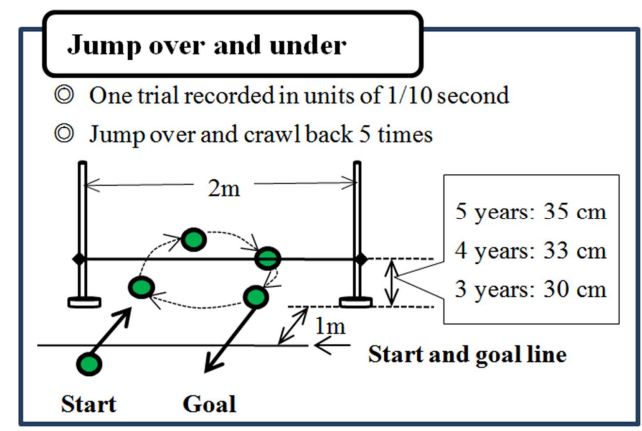

Figure 5. Method of measuring Jump over and under.

\subsubsection{Hanging from A Horizontal Bar}

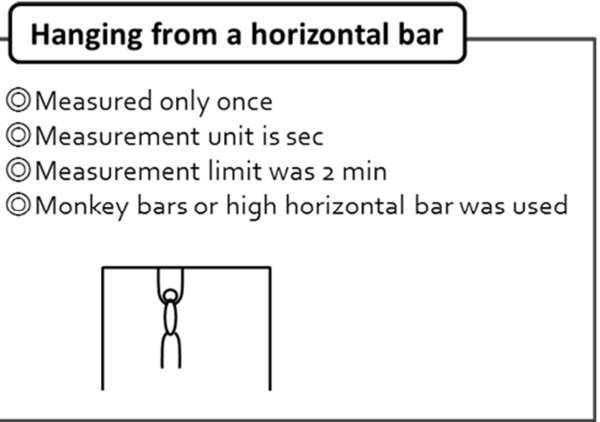

Figure 6. Method of measurement of hanging from a horizontal bar.

\subsubsection{Side Step}

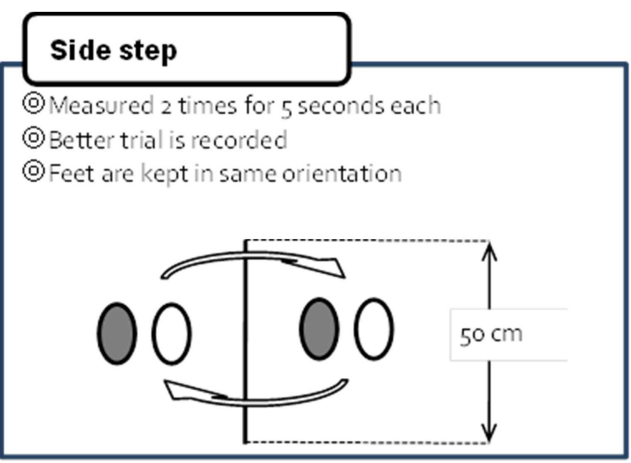

Figure 7. Method of measuring side step.

\subsection{Statistical Analysis}

To investigate whether there were any differences between the girls in the different years, a t-test was performed for both years against the mean values of data obtained in 1999 and 2009. The level of statistical significance was less than $5 \%$.

\section{Results}

\subsection{9 and 2009 Physique and Athletic Ability}

Table 1 shows the statistical values for height and weight, the indicators of physique in this study, and each measure of motor ability in 1999 and 2009. The results of t-tests for physique and motor ability in the two years showed that for 3-, 4-, and 5-year-old girls there were significant differences $(\mathrm{P}<$ $0.05)$ in some items but not in others. The results are shown in Figures 8-16.

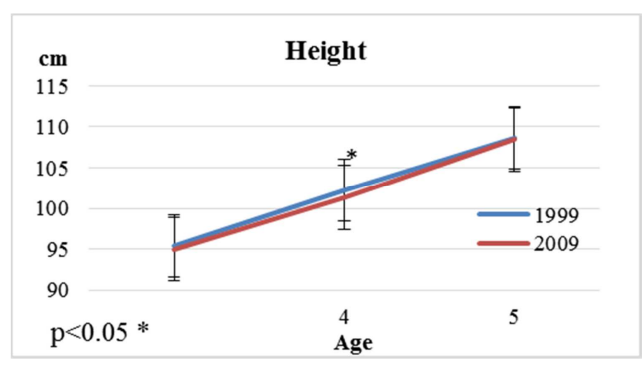

Figure 8. Test of significant difference in height. 


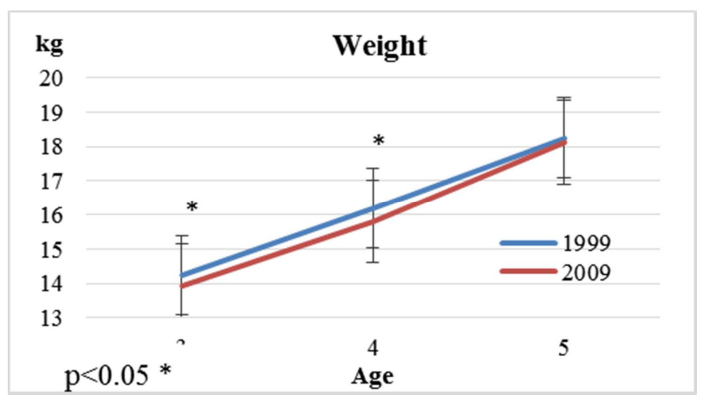

Figure 9. Test of significant difference in weight.

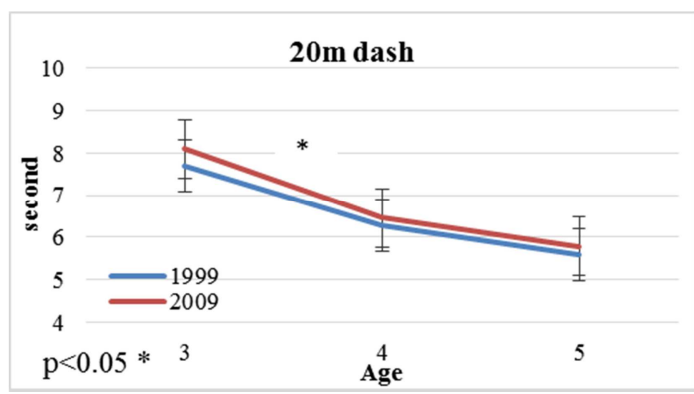

Figure 10. Test of significant difference in $20 \mathrm{~m}$ dash

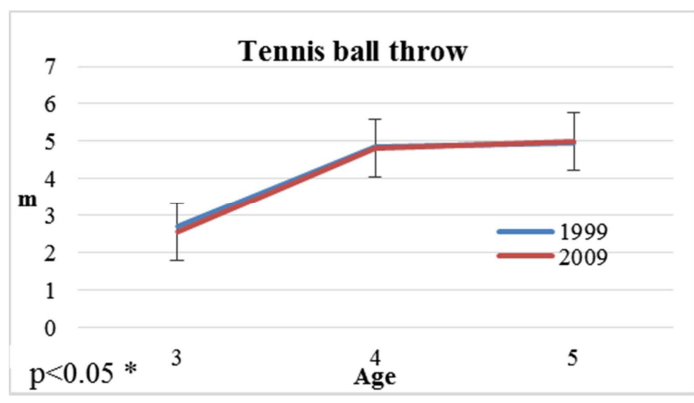

Figure 11. Test of significant difference in tennis ball throw.

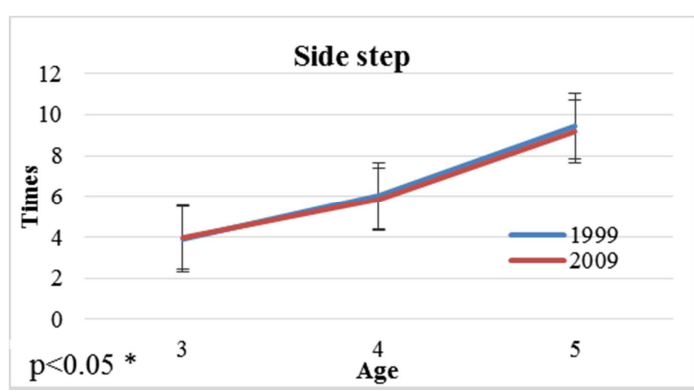

Figure 12. Test of significant difference in side step.

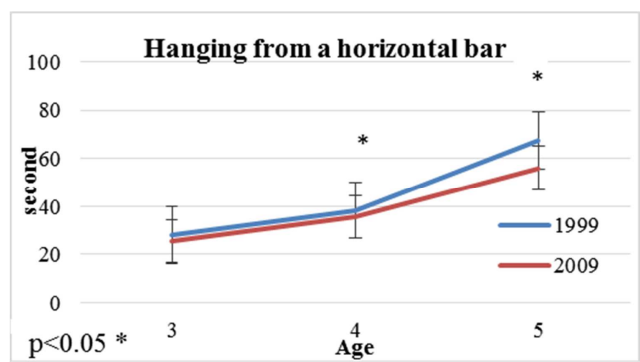

Figure 13. Test of significant difference in hanging from a horizontal bar.

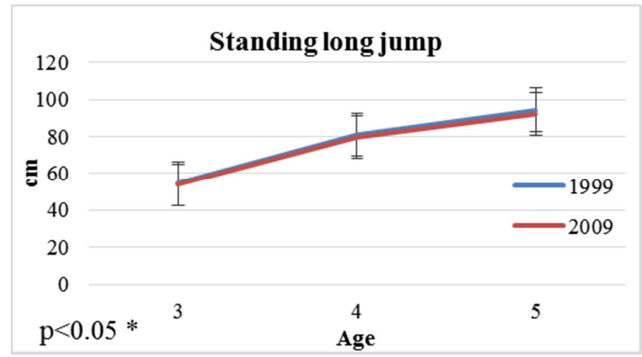

Figure 14. Test of significant difference in standing long jump.

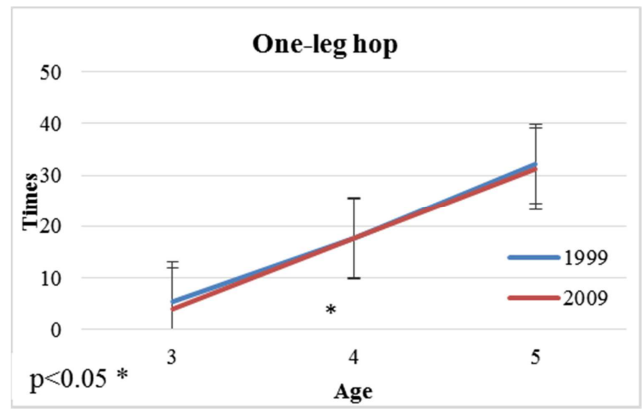

Figure 15. Test of significant difference in one-leg hop.

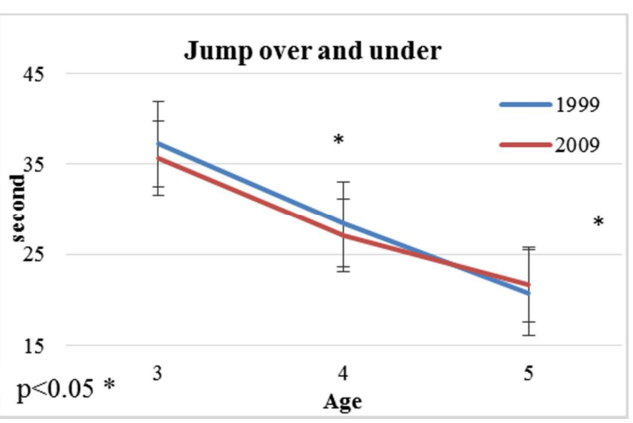

Figure 16. Test of significant difference in jump over and under.

Table 1. Physique Motor skill statistics of Preschool Girls.

\begin{tabular}{llllllll}
\hline \multirow{2}{*}{ Girls } & \multirow{2}{*}{ Year } & \multicolumn{2}{c}{ 3 years old } & \multicolumn{2}{c}{ 4 years old } & \multicolumn{2}{c}{ 5 years old } \\
\cline { 3 - 8 } & & Mean & N & Mean & N & Mean & N \\
\hline \multirow{2}{*}{ Height } & 1999 & 95.38 & 455 & 102.22 & 697 & 108.66 & 738 \\
& 2009 & 95.01 & 170 & 101.37 & 364 & 108.47 & 842 \\
\multirow{2}{*}{ Weight } & 1999 & 14.24 & 456 & 16.19 & 698 & 18.26 & 738 \\
\multirow{2}{*}{20 m dash } & 2009 & 13.92 & 168 & 15.81 & 364 & 18.13 & 842 \\
\multirow{2}{*}{ Tennis ball throw } & 1999 & 7.69 & 409 & 6.29 & 659 & 5.60 & 723 \\
\multirow{2}{*}{ Side step } & 2009 & 8.10 & 168 & 6.46 & 360 & 5.78 & 825 \\
& 1999 & 2.70 & 396 & 4.86 & 154 & 4.97 & 660 \\
& 2009 & 2.56 & 159 & 4.82 & 600 & 5.00 & 823 \\
\end{tabular}




\begin{tabular}{llllllll}
\hline \multirow{2}{*}{ Girls } & \multirow{2}{*}{ Year } & 3 years old & & 4 years old & & 5 years old \\
\cline { 2 - 8 } & & Mean & N & Mean & N & Mean & N \\
\hline \multirow{2}{*}{ Hanging from a horizontal bar } & 1999 & 28.08 & 394 & 38.40 & 668 & 67.49 & 711 \\
\multirow{3}{*}{ Standing long jump } & 2009 & 25.61 & 133 & 35.52 & 343 & 56.06 & 805 \\
\multirow{3}{*}{ One-leg hop } & 1999 & 54.38 & 404 & 81.04 & 660 & 94.32 & 730 \\
& 2009 & 53.76 & 160 & 79.68 & 355 & 92.24 & 829 \\
\multirow{2}{*}{ Jump over and under } & 1999 & 5.39 & 394 & 17.68 & 606 & 32.11 & 711 \\
& 2009 & 3.87 & 133 & 17.67 & 341 & 31.24 & 815 \\
& 1999 & 37.23 & 354 & 28.34 & 579 & 20.80 & 21 \\
\end{tabular}

\subsection{Comparison of Physique in 1999 and 2009}

In 2009 , height was about $0.9 \mathrm{~cm}$ shorter $(\mathrm{P}<0.05)$ in 4 -year-old girls and weight was about $0.3 \mathrm{~kg}$ lighter $(\mathrm{P}<0.05)$ in 3- and 4-year-old girls than in 1999.

\subsection{Comparison of Motor Ability in 1999 and 2009}

The results of a t-test for the $20 \mathrm{~m}$ dash showed significant differences $(\mathrm{P}<0.05)$ for 3-, 4-, and 5-year-old girls. In the tennis ball throw and side step, no significant differences were seen for any age. In hanging from a horizontal bar, significant differences $(P<0.05)$ were seen in $4-$ and 5 -year-old girls. In one-leg hop, a significant difference was seen in 3-year-old girls $(P<0.05)$. In the jump over and under, significant differences $(\mathrm{P}<0.05)$ were seen in all ages.

\section{Discussion}

\subsection{Secular Trend in Physique}

This study used height and weight as indicators of physique, and the results showed significant differences in height and weight between 1999 and 2009 in 4-year-old girls but no significant differences in the other ages. Thus, looking at this 10-year span we may say that there was little change in physique. According to a school health statistics survey [13, 14] on the increasing size of physiques, no marked change has been seen in preschool age children since the late 1980s. The same flat trend is seen in adolescents. Although significant differences were seen in mean values in this study, there have been repeated increases and decreases in recent years and it is thought that the increasing size of the physiques of girls has stalled. Considering this result together with the school health statistics survey [13, 14], one may conclude that the increase in the size of girls' physiques has stopped in recent years.

\subsection{Secular Trend in Motor Ability}

From the results of a previous study, Fujii et al. [11] reported rising trends in the $20 \mathrm{~m}$ dash and standing long jump and a declining trend in the tennis ball throw. In this study, the results of a t-test of the $20 \mathrm{~m}$ dash in 1999 and 2009 showed significant differences in 3-and 5-year-old girls, and running ability in particular is thought to have shown a marked decline over the span of this decade. In the tennis ball throw, no significant difference was found in the results of a t-test comparing 1999 and 2000, and one may conclude that there was no change. In the side step, no significant difference was seen in any of the ages in the results of a t-test comparing 1999 and 2000, from which it may be concluded that there has been no change. In hanging from a horizontal bar, significant differences were seen in 4- and 5-year-old girls, and upper body strength is thought to have declined over this 10 -year span. In the one-leg hop a significant difference was seen in 3-year-olds, while in the jump over and under a significant difference was seen in all ages. Decreased results were seen in the one-leg hop and hanging from a horizontal bar, activities that require more muscle strength. Given no change in physique, this result would show a decline in muscle strength relative to physique. In contrast, in the jump over and under and hanging from a horizontal bar, activities which require relatively more dexterity, better results were seen in 2009. Fujii et al. [11] found that young children were maturing at an earlier age, and the rising trend in the jump over and under and hanging from a horizontal bar, activities that require relatively more dexterity among the physical fitness and motor ability results in this study, is thought to be affected by this earlier maturation. In the $20 \mathrm{~m}$ dash, for which Fujii et al. [11] found an improving trend up to 1999 , a declining or steady trend was seen in this study. Declining or steady trends have also been shown in other studies. With regard to the decline in lower body muscle strength, Nakano et al. [15] studied the number of steps walked per day in young children and showed that the mean number of steps per day had decreased from about 30 years ago, indicating a decrease in physical activity in today's young children. The decrease in mean number of steps is thought to be directly related to leg muscle strength, and the steady or slightly declining trend seen in muscle power of the lower body may be a factor in a continuing decline. The marked decline seen in this study in the $20 \mathrm{~m}$ dash and one-leg hop, activities that require leg strength, may be viewed the same as in the earlier studies of Fujii et al. [11] and Nakano et al. [15]. Ultimately, as shown by Hayakawa and Fujii [4-6], a physique and motor ability suited to the circumstances of the times may be the level of physical fitness that is necessary and valid in future surveys of motor ability in young children.

\section{Conclusion}

This study investigated the trends in physical ability of young girls over the 10-year span from 1999 to 2009 in an attempt to understand recent trends in physique and motor ability of young girls. No fundamental changes were seen 
from the results of this study, although a slight change in physique was seen over this 10-year span. In motor ability, an improving trend was seen in hanging from a horizontal bar, an activity that requires relatively more dexterity. This was thought to be affected by earlier maturation. However, declines were seen in the results for the $20 \mathrm{~m}$ dash, one-leg hop, and hanging from a horizontal bar, from which there is thought to be a decline in running ability and muscle strength in the recent physical fitness trends for young girls.

\section{References}

[1] N. Nishijima, "Tendency of teenagers to decline their physical fitness,” Physical Education Science, Vol. 52, pp. 49-58, 2002.

[2] N. Ueda, "Meaning to live," Iwanami Shoten, 2005.

[3] Ministry of Education, Culture, Sports, Science and Technology, years school health statistics survey http://www.mext.go.jp/sports/b_menu/toukei/kodomo/zencyo/ 1401184.htm, 2017.

[4] K. Hayakawa, K. Fujii, K. Kasuya, T. Kndoh, N. Tanaka, "Exporing appropriate physical fitness for corporate employees derived from the balance of body composition," production Management, Vol. 23 (2), pp. 97-102, 2016.

[5] K. Hayakawa, K. Fujii, K. Kasuya, N. Tanaka, "Optimum validity of the body in defense personnel productivity of defence education judged from physical strength," Production Management, Vol. 24 (1), pp. 69-74, 2017.

[6] K. Hayakawa, K. Fujii, T. Ishigaki, Y. Naito, "Validity Physical Strength Derived from the Secular Span Evaluation in Female University Students", Society for Standardization Studies, Vol. 16 (1), pp. 87-101, 2018.

[7] T. Akimaru, S. Nonaka, T. Hanai, T. Murase, K. Fujii, "Changes of 30 years of childhood development and physical exercise development in Aichi prefecture and their problems,"(for child rearing support), Technical Committee on physical Development, pp. 1-51, 2002.

[8] T. Akimaru, "Changes in the physique and exercise capacity of an infant for 30 years and its problems in child", Growth and Development Study, Vol. 1 (2), pp. 128-132, 2003.

[9] K. Fujii, T. Akimaru, T. Hanai, T. Murase, K. Kasuga, "A study on the age-related changes in physique and athletic ability of young children -Analysis of girls by polynomial-", The Journal of Education and Health Science, Vol. 50 (4), pp. 217-228, 2005.

[10] K. Fujii, T. Akimaru, T. Hanai, T. Murase, T. Sakai, “A study on the age -related changes in physique and athletic ability of infants by Polynomial-Analysis on boys-", Tokai Health and Physical Education, Vol. 27, pp. 9-20, 2005.

[11] K. Fujii, T. Akimaru, T. Hanai, T. Sakai, "Verification on annual change in growth and development of physique and exercise capacity of an infant -Approach from the viewpoint of physical maturity-," The Japanese Journal of Physical Fitness and Sports Medicine, Vol. 55, pp. 489-502, 2006.

[12] K. Kasuga, "Longitudinal transition of physical strength difference in early childhood Based on follow- up data for 3 years," Growth and Development Study", Vol. 41, pp. 17-27, 2009.

[13] http://www.mext.go.jp/b_menu/toukei/chousa05/hoken/kekka/ k_detail/1345146.htm, Ministry of Education, Culture, Sports, Science and Technology, years school health statistics survey, 2013.

[14] http://www.mext.go.jp/b_menu/toukei/chousa04/tairyoku/kek $\mathrm{ka} / \mathrm{k}$ detail/1340101.htm, Ministry of Education, Culture, Sports, Science and Technology years school health statistics survey, 2014.

[15] T. Nakano, K. Kasuga, T. Murase, "Study of appropriate amount of physical activity in young children considering relationship with lifestyle and physical fitness," Growth and Development Study, Vol. 46, pp. 49-58, 2010. 